\section{New Theory}

ISSN: 2149-1402
37 (2021) 45-57

Journal of New Theory

https://dergipark.org.tr/en/pub/jnt

Open Access

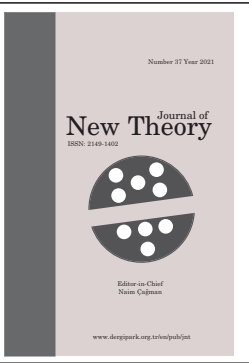

\title{
A New Method to Obtain PH-Helical Curves in $\mathbb{E}^{n+1}$
}

\author{
Ahmet Mollaoğulları ${ }^{1}$ (D), Mehmet Gümüşs $^{2}$ (D), Kazım İlarslan $^{3}$ (D) Çetin Camcı $^{4}$ (D)
}

\author{
Article History \\ Received: 23 Nov 2021 \\ Accepted: 26 Dec 2021 \\ Published: 31 Dec 2021 \\ 10.53570/jnt.1027564 \\ Research Article
}

\begin{abstract}
Helical curves are constructed by the property that their unit tangents make a constant angle with a chosen constant direction. There are relations between polynomial planar curves, helices and Pythagorean-hodograph or shortly PH-curves. The aim of this paper is to obtain a method which generate $\mathrm{PH}$-curves and $\mathrm{PH}$-helical curves from a planar curve in Euclidean Space $\mathbb{E}^{n+1}$. Furthermore, some examples are given in $\mathbb{E}^{4}$ and $\mathbb{E}^{5}$ to explain the method neatly.
\end{abstract}

Keywords - PH-curve, helical curve, PH-helical curve, planar curve, polynomial curve

Mathematics Subject Classification (2020) - 53A04, 53A05

\section{Introduction}

Helix is an interesting curve which has been studied by many mathematicians in differential geometry. We can see helices or its various types of general helix in many areas such as nature, physics, kinematic motion, design of architectural building and the structure of the DNA. A curve is a general helix (or constant slope curve) if its tangent vector field makes a constant angle with a fixed straight line in Euclidean space $\mathbb{E}^{3}$. In 1802, Lancret [1] introduced the famous result on helices and it was proved in 1845 by B. de Saint Venant that the ratio of its curvature to torsion is constant. Indeed, helix is a geometric curve whose curvature and torsion are non-vanishing constants [2]. In addition, if the curvature and the torsion of a curve are non-zero constants then the curve is a general helix. So it is clear that helix is a special case of general helix. Furthermore, straight lines and circles are degenerate-helices.

In the curve theory, another important curves are Pythagorean-Hodograph curves, or shortly PHcurves. These curves have many applications such as CNC machining, offseting, computer aided geometric design and motion planning. They were first introduced in Farouki et al. [3]. Because they are characterized by their arclength which is a polynomial function, they have attracted the attention of researchers and have been widely studied in [4-11].

In [12], Izumiya and Takeuchi show that cylindrical helices can be obtained from planar curves. They give a method to obtain a helical curve from a planar curve in their study. Moreover, there are well-known two methods for constructing PH-curves: One of them is by using complex numbers representation [4] and the other method is quaternion representation [5,7]. Via the point of view

\footnotetext{
${ }^{1}$ ahmet_m@comu.edu.tr (Corresponding Author); $\quad{ }^{2}$ mehmetgumus@comu.edu.tr; $\quad{ }^{3}$ kilarslan@yahoo.com, ${ }^{4}$ ccamci@comu.edu.tr

${ }^{1,4}$ Department of Mathematics, Faculty of Arts and Sciences, Onsekiz Mart University, Çanakkale, Turkey

${ }^{2}$ Department of Accounting and Tax Treatment, Lapseki Vocational School, Onsekiz Mart University, Çanakkale, Turkey

${ }^{3}$ Department of Mathematics, Faculty of Arts and Sciences, Kırıkkale University, Kırıkkale, Turkey
} 
of these studies Camcı and İlarslan give a new method for construction of PH-helical curves in 3dimensional Euclidean space in [13]. In this paper, we extend their theory to the Euclidean space $\mathbb{E}^{n+1}$.

\section{Basic Concepts and Notions}

Let $\alpha: I \subset \mathbb{R} \rightarrow \mathbb{E}^{n}$ be a regular curve in Euclidean space $\mathbb{E}^{n}$. It is well known that the curve $\alpha$ is said to be of unit speed (or parameterized by arclength function $s$ ) if $\left\langle\alpha^{\prime}(s), \alpha^{\prime}(s)\right\rangle=1$, where $\langle$,$\rangle is$ the standard inner product of $\mathbb{E}^{n}$ given by

$$
\langle X, Y\rangle=\sum_{i=1}^{n} x_{i} y_{i},
$$

where $X=\left(x_{1}, x_{2}, \ldots, x_{n}\right), Y=\left(y_{1}, y_{2}, \ldots, y_{n}\right) \in \mathbb{E}^{n}$. Let $\left\{V_{1}, V_{2}, \ldots, V_{n}\right\}$ be the moving Frenet frame along a space curve $\alpha$, where $V_{i}(i=1,2, \ldots, n)$ denote ith Frenet vector field of $\alpha$. Then, the Frenet formulas are given by

$$
\left\{\begin{array}{l}
V_{1}^{\prime}(t)=\nu(t) k_{1}(t) V_{2}(t) \\
V_{i}^{\prime}(t)=\nu(t)\left(-k_{i-1}(t) V_{i-1}(t)+k_{i}(t) V_{i+1}(t)\right), \quad 2 \leq i \leq n-1 \\
V_{n}^{\prime}(t)=-\nu(t) k_{n-1}(t) V_{n-1}(t)
\end{array}\right.
$$

where $\nu(t)=\left\|\alpha^{\prime}(t)\right\|$ and $k_{i}(i=1,2, \ldots, n-1)$ denote the i-th curvature function of the curve (see $[2,14])$. If the curve lies in a hyperplane of $\mathbb{E}^{n}$, then it is said that $\alpha$ is a $(n-1)$-flat curve. It is well known that $\alpha$ is $(n-1)$-flat curve in $\mathbb{E}^{n}$ if and only if $k_{n-1}(t)=0$ [15]. Harmonic curvature functions were defined by Özdamar and Hacisalihoğlu in [16] as follow:

Definition 2.1. Let $\alpha$ be a regular curve in $\mathbb{E}^{n}$. Harmonic curvatures of the curve $\alpha$ are defined by $H_{i}: I \subset \mathbb{R} \rightarrow \mathbb{R}$ such that

$$
H_{i}= \begin{cases}0, & i=1 \\ \frac{k_{1}}{k_{2}}, & i=2 \\ \left\{V_{1}\left[H_{i-1}\right]+k_{i} H_{i-2}\right\} \frac{1}{k_{i+1}}, & i=3,4, \ldots, n-2\end{cases}
$$

Characterizations for generalized helices by using the harmonic curvatures of the curve were studied by Camc1 et al. in [17]. They obtained some important results for generalized helix in $n$-dimensional Euclidean space $\mathbb{E}^{n}$.

Theorem 2.2. [17] Let $\alpha$ be a non-degenerate curve in n-dimensional Euclidean space $\mathbb{E}^{n}$. Let $\left\{V_{1}, V_{2}, \ldots, V_{n}\right\}$ and $\left\{H_{1}, H_{2}, \ldots, H_{n-2}\right\}$ be the Frenet frame and harmonic curvatures of the curve, respectively. Then, $\alpha$ is a general helix if and only if $H_{n-2}^{\prime}+\nu k_{n-1} H_{n-3}=0$.

Definition 2.3. [17] Let $\alpha$ be a unit speed, non-degenerate curve in n-dimensional Euclidean space $\mathbb{E}^{n}$. Let $\left\{V_{1}, V_{2}, \ldots, V_{n}\right\}$ and $\left\{H_{1}, H_{2}, \ldots, H_{n-2}\right\}$ be the Frenet frame and harmonic curvatures of the curve, respectively. The vector

$$
D=V_{1}+H_{1} V_{3}+\ldots+H_{n-2} V_{n}
$$

is called the generalized Darboux vector of the curve $\alpha$.

The relationship between the generalized Darboux vector $D$ and the general helix is given by the following theorem. 
Theorem 2.4. [17] Let $\alpha$ be a unit speed, non-degenerate curve in Euclidean space $\mathbb{E}^{n}$. Let $\left\{V_{1}, V_{2}, \ldots, V_{n}\right\}$ and $\left\{H_{1}, H_{2}, \ldots, H_{n-2}\right\}$ be the Frenet frame and harmonic curvatures of the curve, respectively. Then $\alpha$ is a generalized helix if and only if $D$ is a constant vector.

Definition 2.5. [3] Let $\alpha: I \subset \mathbb{R} \rightarrow \mathbb{E}^{n+1}(t \rightarrow \alpha(t))$ be a polynomial curve. If the speed of the curve $v(t)=\|\dot{\alpha}(t)\|$ is polynomial, then it is called a Pythagorean-hodograph or shortly PH-curve.

One of the well-known equations in mathematics history is the Pythagorean equation, i.e., $a^{2}+b^{2}=$ $c^{2}$. General solution of this equation is [18] :

$$
a=t\left(u^{2}-v^{2}\right), \quad b=2 t u v, \quad c=t\left(u^{2}+v^{2}\right)
$$

where $\mathrm{t}$ is a scale parameter. It can be deduced from Equation (2) that rational solution of the Pythagorean n-tuple $a_{1}^{2}+a_{2}^{2}+\ldots+a_{n}^{2}=\sigma^{2}$ can be given as follows:

$$
\begin{aligned}
a_{1} & =t\left(u_{1}^{2}-u_{2}^{2}-\ldots-u_{n}^{2}\right) \\
a_{i} & =2 t a_{1} a_{i}, i=2,,, n \\
\sigma & =t\left(u_{1}^{2}+u_{2}^{2}+\ldots+u_{n}^{2}\right)
\end{aligned}
$$

where $u_{i}(i=1,2, \ldots, n)$ are integers and $t$ is scaling parameter.

\section{The Construction of a Helix in $\mathbb{E}^{n+1}$}

In Euclidean space $\mathbb{E}^{n+1}$, let $H^{n}$ be a $n$-hyperplane and $\gamma: I \rightarrow H^{n} \subset \mathbb{E}^{n+1}$ be a regular curve. Hence, we can define a curve such that

$$
\beta(t)=\gamma(t)+\left(\cot \theta \int_{0}^{t}\|\dot{\gamma}(u)\| d u\right) \vec{a}+\vec{c}
$$

where $\|\vec{a}\|=1,\langle\vec{a}, \dot{\gamma}(t)\rangle=0, \theta \in \mathbb{R}, \vec{a}$ and $\vec{c}$ is a constant vector. Therefore, from Equation (3),

$$
\dot{\beta}(t)=\dot{\gamma}(t)+(\cot \theta\|\dot{\gamma}(t)\|) \vec{a}
$$

and from Equation (4),

$$
\|\dot{\beta}(t)\|=\frac{1}{\sin \theta}\|\dot{\gamma}(t)\|
$$

Thus, from Equations (4) and (5), we have

$$
T_{\beta}=\sin \theta T_{\gamma}+\cos \theta \vec{a},\left\langle T_{\beta}, \vec{a}\right\rangle=\cos \theta
$$

where $T_{\beta}$ (resp. $T_{\gamma}$ ) is a tangent of the curve $\beta$ (resp. $\gamma$ ). From Equation (6), it can be observed that $\beta$ is a helix.

Theorem 3.1. All helix curves in Euclidean space $\mathbb{E}^{n+1}$ can be obtained from a regular curve which lies in a hyperplane $H^{n} \subset \mathbb{E}^{n+1}$.

Proof. Let $\beta: I \rightarrow \mathbb{E}^{n+1}$ be a unit speed helix curve. Hence, the generalized Darboux vector $D(s)$ of the curve $\beta$ is a constant where

$$
D(s)=V_{1}+H_{1} V_{3}+. .+H_{n-1} V_{n+1}
$$

In this case, unit axes of the curve $\beta$ is equal to

$$
\vec{a}=\frac{D(s)}{\|D(s)\|}=\frac{V_{1}+H_{1} V_{3}+. .+H_{n-1} V_{n+1}}{\sqrt{1+H_{1}^{2}+H_{2}^{2}+. .+H_{n-1}^{2}}}
$$


and we have $\left\langle T_{\beta}, \vec{a}\right\rangle=\cos \theta$ where $\beta^{\prime}(s)=T_{\beta}$. Hence we can see that

$$
\left\langle\beta^{\prime}, \vec{a}\right\rangle=\cos \theta
$$

If we consider the curve

$$
\gamma(s)=\beta(s)-\langle\beta(s), \vec{a}\rangle \vec{a},
$$

then we have $\langle\gamma(s), \vec{a}\rangle=0$. This means that the curve $\gamma$ lies in $H^{n}$. Moreover, we can see that

$$
\left\|\gamma^{\prime}(s)\right\|=\left\|\beta^{\prime}(s)-\left\langle\beta^{\prime}(s), \vec{a}\right\rangle \vec{a}\right\|=\sin \theta
$$

From Equations (8) and (9), we have

$$
\gamma(s)+\left(\cot \theta \int_{0}^{s}\|\dot{\gamma}(u)\| d u\right) \vec{a}=\beta(s)-s \cos \theta \vec{a}+s \cot \theta \sin \theta \vec{a}=\beta(s)
$$

From Equation (5), we can see that if the curve $\alpha$ is a polynomial curve, then the curve $\beta$ is a polynomial curve. Hence, from Theorem 3.1, we can give following theorem.

Theorem 3.2. Under the above notation, if the curve $\alpha$ is a PH-curve, then the curve $\beta$ is a PH-helical curve. Moreover, all PH-helical curves in Euclidean space $\mathbb{E}^{n+1}$ can be obtained from a PH-curve $\gamma: I \rightarrow H^{n} \subset \mathbb{E}^{n+1}$

The following theorems are given in [13] for obtaining PH-curves and PH-helical curves from planar curves in $\mathbb{E}^{3}$.

Theorem 3.3. [13] Let $H^{2}(a, b, c)=\left\{\left(p_{1}, p_{2}, p_{3}\right) \in \mathbb{E}^{3}: a p_{1}+b p_{2}+c p_{1}+d=0\right\}$ be a plane in $\mathbb{E}^{3}$. If the curve $\gamma: I \rightarrow H^{2}, \gamma(t)=\left(\gamma_{1}(t), \gamma_{2}(t), \gamma_{3}(t)\right)$ is a PH-curve, then

$$
\begin{aligned}
& \gamma_{1}(t)=\int\left(b \sqrt{a^{2}+b^{2}+c^{2}}\left(u^{2}(t)-v^{2}(t)\right)-2 a c u(t) v(t)\right) d t \\
& \gamma_{2}(t)=\int\left(-a \sqrt{a^{2}+b^{2}+c^{2}}\left(u^{2}(t)-v^{2}(t)\right)-2 b c u(t) v(t)\right) d t \\
& \gamma_{3}(t)=\int 2\left(a^{2}+b^{2}\right) u(t) v(t) d t \\
& \sigma(t)=\sqrt{a^{2}+b^{2}} \sqrt{a^{2}+b^{2}+c^{2}}\left(u^{2}(t)+v^{2}(t)\right)
\end{aligned}
$$

where $u(t)$ and $v(t)$ are polynomials and

$$
\sigma^{2}(t)=\left(\gamma_{1}^{\prime}(t)\right)^{2}+\left(\gamma_{2}^{\prime}(t)\right)^{2}+\left(\gamma_{3}^{\prime}(t)\right)^{2}
$$

Theorem 3.4. [13] Let $H^{2}(a, b, c)$ be a plane in $\mathbb{E}^{3}$ where

$$
H^{2}(a, b, c)=\left\{\left(p_{1}, p_{2}, p_{3}\right): a p_{1}+b p_{2}+c p_{3}+d=0\right\}
$$

In this case, we get all PH- helical curves from planes $H^{2}(a, b, c)$ in $\mathbb{E}^{3}$ such that

$$
\gamma_{(a, b, c)}(\gamma, t)=\beta(t)=\left(\beta_{1}(t), \beta_{2}(t), \beta_{3}(t)\right)
$$

where

$$
\begin{gathered}
\beta_{1}(t)=\int\left[b \sqrt{a^{2}+b^{2}+c^{2}}\left(u^{2}(t)-v^{2}(t)\right)-2 a c u(t) v(t)+a\left(a^{2}+b^{2}\right) \cot \theta\left(u^{2}(t)+v^{2}(t)\right)\right] d t \\
\beta_{2}(t)=\int\left(-a \sqrt{a^{2}+b^{2}+c^{2}}\left(u^{2}(t)-v^{2}(t)\right)-2 b c u(t) v(t)+b\left(a^{2}+b^{2}\right) \cot \theta\left(u^{2}(t)+v^{2}(t)\right)\right) d t \\
\beta_{3}(t)=\int\left[2\left(a^{2}+b^{2}\right) u(t) v(t)+c\left(a^{2}+b^{2}\right) \cot \theta\left(u^{2}(t)+v^{2}(t)\right)\right] d t
\end{gathered}
$$

Hence, axis of the curve $\beta$ is equal to

$$
\vec{a}=\left(\frac{a}{\sqrt{a^{2}+b^{2}+c^{2}}}, \frac{b}{\sqrt{a^{2}+b^{2}+c^{2}}}, \frac{c}{\sqrt{a^{2}+b^{2}+c^{2}}}\right)
$$




\section{PH-Helical Curves in $\mathbb{E}^{n+1}$}

In this section we give a method to obtain a $\mathrm{PH}$ curve in $H^{n} \subset \mathbb{E}^{n+1}$. Then, we give a theorem in $\mathbb{E}^{4}$ for the given method. After then, using Theorem 3.2, we give a theorem to construct a PH-helical curve in $\mathbb{E}^{4}$. Finally, we support these theorems by some examples.

For $a_{1}, a_{2}, \ldots, a_{n+1}, a_{n+2} \in \mathbb{R}$, let

$$
H^{n}\left(a_{1}, a_{2}, \ldots, a_{n+1}\right)=\left\{\left(p_{1}, p_{2}, \ldots, p_{n+1}\right) \in \mathbb{E}^{n+1} \mid \sum_{i=1}^{n+1}\left(a_{i} p_{i}\right)+a_{n+2}=0\right\}
$$

be a hyperplane of $\mathbb{E}^{n+1}$. Assume that $\gamma: I \rightarrow H^{n}\left(a_{1}, a_{2}, \ldots, a_{n+1}\right), \gamma(t)=\left(\gamma_{1}(t), \gamma_{2}(t), \ldots, \gamma_{n+1}(t)\right)$ is a $P H-$ curve with the speed of a polynomial such as $\sigma(t)$. Therefore, we have

$$
\sum_{i=1}^{n+1}\left(\gamma_{i}^{\prime}(t)\right)^{2}=\sigma^{2}(t)
$$

Since $\gamma$ lies in $H^{n}\left(a_{1}, a_{2}, \ldots, a_{n+1}\right)$ we write

$$
\sum_{i=1}^{n+1}\left(a_{i} \gamma_{i}(t)\right)+a_{n+2}=0
$$

From Equation (10), we get

$$
\sum_{i=1}^{n}\left(\frac{\gamma_{i}^{\prime}(t)}{\gamma_{n+1}^{\prime}(t)}\right)^{2}+1=\left(\frac{\sigma(t)}{\gamma_{n+1}^{\prime}(t)}\right)^{2}
$$

Differentiation Equation (11) give us

$$
\sum_{i=1}^{n+1} a_{i} \gamma_{i}^{\prime}(t)=0
$$

Applying the following substitutions

$$
\begin{aligned}
X_{i} & =\frac{\gamma_{i}^{\prime}(t)}{\gamma_{n+1}^{\prime}(t)}, i=1,2, \ldots, n \\
\omega & =\frac{\sigma(t)}{\gamma_{n+1}^{\prime}(t)}
\end{aligned}
$$

we have

$$
\sum_{i=1}^{n}\left(X_{i}^{2}\right)+1=\omega^{2}
$$

and

$$
\sum_{i=1}^{n}\left(a_{i} X_{i}\right)+a_{n+1}=0
$$

From Equation (14), $X_{n}$ can be written as

$$
X_{n}=\sum_{i=1}^{n-1}\left(b_{i} X_{i}\right)+b_{n}
$$

where

$$
b_{i}=-\frac{a_{i}}{a_{n}}, i=1,2, \ldots, n-1
$$


and

$$
b_{n}=-\frac{a_{n+1}}{a_{n}}
$$

Considering Equations (13) and (15), we get

$$
\sum_{i=1}^{n-1}\left(1+b_{i}^{2}\right) X_{i}^{2}+\sum_{\substack{i, j=1 \\ i \neq j}}^{n}\left(b_{i} b_{j} X_{i} X_{j}\right)+\sum_{i=1}^{n} 2\left(b_{i} b_{n+1} X_{i}\right)=\omega^{2}
$$

If we apply the following transformations in Equation (16),

$$
X_{i}=Y_{i}-\frac{b_{i} b_{n+1}}{1+b_{1}^{2}+b_{2}^{2}+\ldots b_{n}^{2}}, i=1,2, \ldots, n
$$

then we find

$$
\sum_{i=1}^{n-1}\left(1+b_{i}^{2}\right) Y_{i}^{2}+\sum_{\substack{i, j=1 \\ i \neq j}}^{n}\left(b_{i} b_{j} Y_{i} Y_{j}\right)+\left(\frac{1+b_{1}^{2}+b_{2}^{2}+\ldots+b_{n}^{2}}{1+b_{1}^{2}+b_{2}^{2}+\ldots+b_{n-1}^{2}}\right)=\omega^{2}
$$

We can rewrite this quadratic form as

$$
Y^{t} A Y+\left(\frac{1+b_{1}^{2}+b_{2}^{2}+\ldots+b_{n}^{2}}{1+b_{1}^{2}+b_{2}^{2}+\ldots+b_{n-1}^{2}}\right)=\omega^{2}
$$

where

$$
Y=\left(\begin{array}{c}
Y_{1} \\
Y_{2} \\
\vdots \\
Y_{n-1}
\end{array}\right) \text { and } A=\left[\begin{array}{cccc}
1+b_{1}^{2} & b_{1} b_{2} & \ldots & b_{1} b_{n-1} \\
b_{1} b_{2} & 1+b_{2}^{2} & \ldots & b_{2} b_{n-1} \\
\vdots & \vdots & \vdots & \vdots \\
b_{1} b_{n-1} & b_{2} b_{n-1} & \ldots & 1+b_{n-1}^{2}
\end{array}\right]
$$

One can calculate the eigenvalues of $\mathrm{A}$ as $\lambda_{1}=1, \lambda_{2}=1, \ldots, \lambda_{n-2}=1, \lambda_{n-1}=1+b_{1}^{2}+b_{2}^{2}+\ldots b_{n-1}^{2}$ and the corresponding eigenvectors of $\mathrm{A}$ as columns of the following matrix:

$$
A=\left[\begin{array}{cccccc}
-\frac{b_{2}}{b_{1}} & -\frac{b_{3}}{b_{1}} & \cdots & -\frac{b_{n-1}}{b_{1}} & -\frac{b_{n-2}}{b_{1}} & \frac{b_{1}}{b_{n-1}} \\
1 & 0 & \cdots & 0 & 0 & \frac{b_{2}}{b_{n-1}} \\
0 & 1 & \cdots & 0 & 0 & \frac{b_{3}}{b_{n-1}} \\
\vdots & \vdots & \ddots & \vdots & \vdots & \vdots \\
0 & 0 & \cdots & 1 & 0 & \frac{b_{n-2}}{b_{n-1}} \\
0 & 0 & \cdots & 0 & 1 & 1
\end{array}\right]
$$

Here $I_{n-2}$ is the Idendity matrix of dimension $(n-2)$. We define the index set

$$
S_{n-j}=\{1, n-j+1, n-j+2, \ldots, n-1\}
$$

so that we can give a general form of the orthonormal matrix $Q$ obtained by eigenvectors of A which diagonalize Equation (17) and satisfies $Z=Q Y$ where 


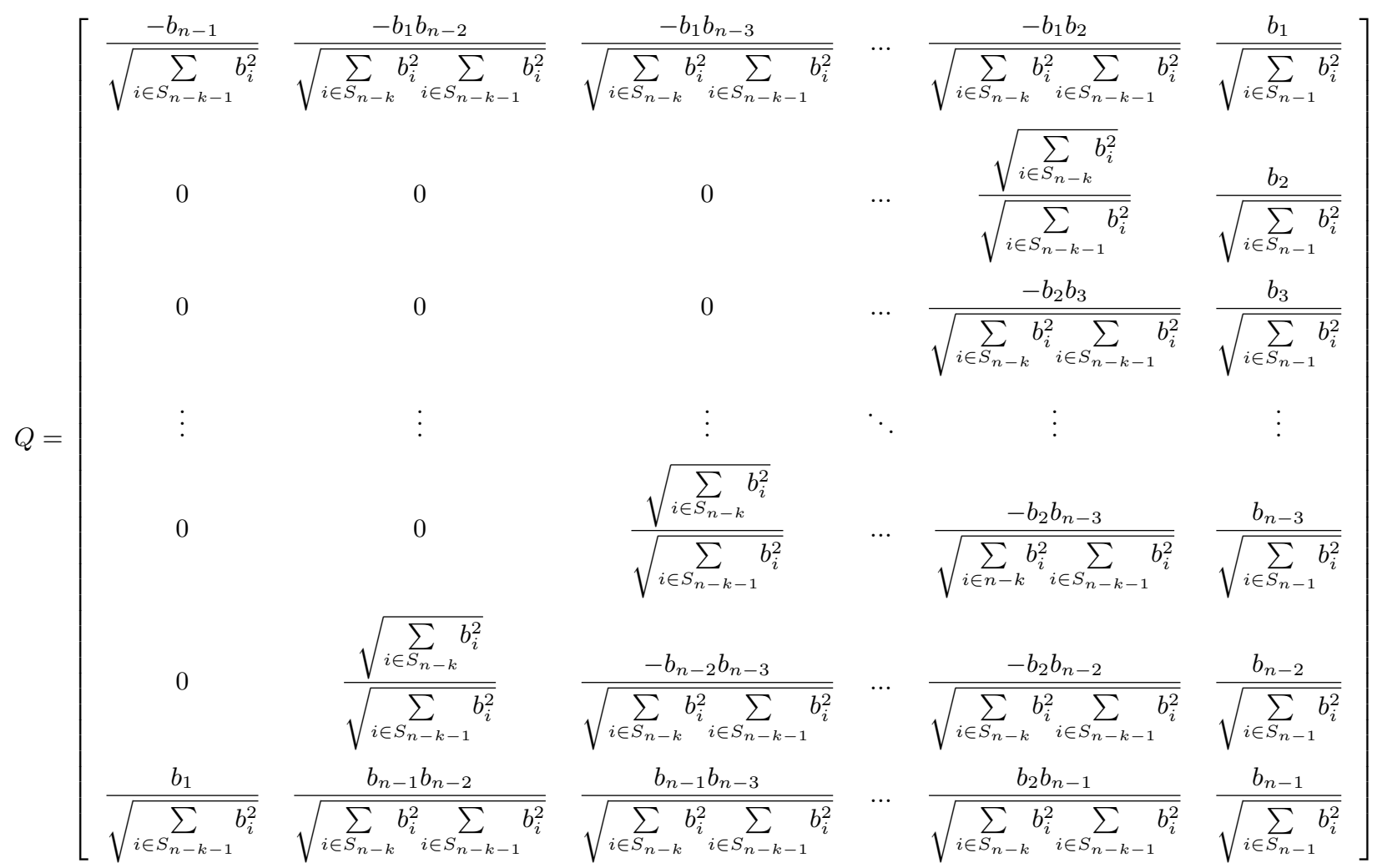

Here $k$ stands for the k-th column of $Q$. To clarify $Q$, we give the following examples for spacial cases $n=3$ and 4 respectively:

$Q=\left[\begin{array}{ccc}\frac{-b_{3}}{\sqrt{b_{1}^{2}+b_{3}^{2}}} & \frac{-b_{1} b_{2}}{\sqrt{b_{1}^{2}+b_{3}^{2}} \sqrt{b_{1}^{2}+b_{2}^{2}+b_{3}^{2}}} & \frac{b_{1}}{\sqrt{b_{1}^{2}+b_{2}^{2}+b_{3}^{2}}} \\ 0 & \frac{\sqrt{b_{1}^{2}+b_{3}^{2}}}{\sqrt{b_{1}^{2}+b_{2}^{2}+b_{3}^{2}}} & \frac{b_{2}}{\sqrt{b_{1}^{2}+b_{2}^{2}+b_{3}^{2}}} \\ \frac{b_{1}}{\sqrt{b_{1}^{2}+b_{3}^{2}}} & \frac{-b_{2} b_{3}}{\sqrt{b_{1}^{2}+b_{3}^{2}} \sqrt{b_{1}^{2}+b_{2}^{2}+b_{3}^{2}}} & \frac{b_{3}}{\sqrt{b_{1}^{2}+b_{2}^{2}+b_{3}^{2}}}\end{array}\right]$

$Q=\left[\begin{array}{cccc}\frac{-b_{4}}{\sqrt{b_{1}^{2}+b_{4}^{2}}} & \frac{-b_{1} b_{3}}{\sqrt{b_{1}^{2}+b_{4}^{2}} \sqrt{b_{1}^{2}+b_{2}^{2}+b_{3}^{2}+b_{4}^{2}}} & \frac{-b_{1} b_{2}}{\sqrt{b_{1}^{2}+b_{3}^{2}+b_{4}^{2}} \sqrt{b_{1}^{2}+b_{2}^{2}+b_{3}^{2}+b_{4}^{2}}} & \frac{b_{1}}{\sqrt{b_{1}^{2}+b_{2}^{2}+b_{3}^{2}+b_{4}^{2}}} \\ 0 & 0 & \frac{\sqrt{b_{1}^{2}+b_{3}^{2}+b_{4}^{2}}}{\sqrt{b_{1}^{2}+b_{2}^{2}+b_{3}^{2}+b_{4}^{2}}} & \frac{b_{2}}{\sqrt{b_{1}^{2}+b_{2}^{2}+b_{3}^{2}+b_{4}^{2}}} \\ 0 & \frac{\sqrt{b_{1}^{2}+b_{4}^{2}}}{\sqrt{b_{1}^{2}+b_{3}^{2}+b_{4}^{2}}} & \frac{-b_{2} b_{3}}{\sqrt{b_{1}^{2}+b_{3}^{2}+b_{4}^{2}} \sqrt{b_{1}^{2}+b_{2}^{2}+b_{3}^{2}+b_{4}^{2}}} & \frac{b_{3}}{\sqrt{b_{1}^{2}+b_{2}^{2}+b_{3}^{2}+b_{4}^{2}}} \\ \frac{-b_{1}}{\sqrt{b_{1}^{2}+b_{4}^{2}}} & \frac{-b_{3} b_{4}}{\sqrt{b_{1}^{2}+b_{4}^{2}} \sqrt{b_{1}^{2}+b_{2}^{2}+b_{3}^{2}+b_{4}^{2}}} & \frac{-b_{2} b_{4}}{\sqrt{b_{1}^{2}+b_{3}^{2}+b_{4}^{2}} \sqrt{b_{1}^{2}+b_{2}^{2}+b_{3}^{2}+b_{4}^{2}}} & \frac{b_{4}}{\sqrt{b_{1}^{2}+b_{2}^{2}+b_{3}^{2}+b_{4}^{2}}}\end{array}\right]$

Applying $Z=Q Y$ in Equation (17) we get

$$
\sum_{i=1}^{n-2}\left(Z_{i}^{2}\right)+\left(1+b_{1}^{2}+b_{2}^{2}+\ldots+b_{n-1}^{2}\right) Z_{n-1}^{2}+\frac{1+b_{1}^{2}+b_{2}^{2}+b_{3}^{2}+\ldots+b_{n}^{2}}{1+b_{1}^{2}+b_{2}^{2}+\ldots+b_{n-1}^{2}}=\omega^{2}
$$


Because Equation (18) is a Pythagorean ( $\mathrm{n}+2)$-tuple, rational solution for this equation is

$$
\begin{aligned}
Z_{1}(t) & =\frac{r(t)}{s(t)}\left(u_{1}^{2}(t)-\sum_{i=2}^{n-1} u_{i}^{2}(t)\right) \\
Z_{i}(t) & =\frac{2 r(t)}{s(t)} u_{1}(t) u_{i}(t), \quad i=2,3, \ldots, n-1 \\
\sqrt{\frac{1+b_{1}^{2}+b_{2}^{2}+b_{3}^{2}+\ldots+b_{n}^{2}}{1+b_{1}^{2}+b_{2}^{2}+\ldots+b_{n-1}^{2}}} & =\frac{2 r(t)}{s(t)} u_{1}(t) u_{n}(t) \\
\omega(t) & =\frac{r(t)}{s(t)} \sum_{i=1}^{n} u_{i}^{2}(t)
\end{aligned}
$$

where $u_{1}(t), u_{2}(t), \ldots, u_{n}(t)$ are arbitrary polynomials and

$$
s(t)=\frac{2 \sqrt{1+b_{1}^{2}+b_{2}^{2}+\ldots+b_{n}^{2}}}{\sqrt{1+b_{1}^{2}+b_{2}^{2}+b_{3}^{2}+\ldots+b_{n+1}^{2}}} r(t) u_{1}(t) u_{n}(t)
$$

Hence, the components of $(\gamma)$ can be written with arbitrary polynomials $u_{1}(t), u_{2}(t), \ldots, u_{n}(t)$ and the reals $a_{1}, a_{2}, \ldots, a_{n+1}$.

For $n=3$, we give the following theorem in $\mathbb{E}^{4}$.

Theorem 4.1. For the hyperplane,

$$
H^{3}\left(a_{1}, a_{2}, a_{3}, a_{4}\right)=\left\{\left(p_{1}, p_{2}, p_{3}, p_{4}\right): a_{1} p_{1}+a_{2} p_{2}+a_{3} p_{3}+a_{4} p_{4}+a_{5}=0, p_{1}, p_{2}, p_{3}, p_{4} \in \mathbb{R}\right\}
$$

let $\gamma: I \rightarrow H^{3}, \gamma(t)=\left(\gamma_{1}(t), \gamma_{2}(t), \gamma_{3}(t), \gamma_{4}(t)\right)$ lies in $H^{3}$. Then, $\gamma$ is a PH-curve in $\mathbb{E}^{4}$ with the followings:

$$
\begin{gathered}
\gamma_{1}=\int\left(\begin{array}{c}
a_{2}\left(a_{1}^{2}+a_{2}^{2}+a_{3}^{2}\right) \sqrt{a_{1}^{2}+a_{2}^{2}+a_{3}^{2}+a_{4}^{2}}\left(u_{1}^{2}-u_{2}^{2}-u_{3}^{2}\right) \\
-2 a_{1} a_{3} \sqrt{a_{1}^{2}+a_{2}^{2}+a_{3}^{2}} \sqrt{a_{1}^{2}+a_{2}^{2}+a_{3}^{2}+a_{4}^{2}} u_{1} u_{2}-2 a_{1} a_{4} \sqrt{a_{1}^{2}+a_{2}^{2}} \sqrt{a_{1}^{2}+a_{2}^{2}+a_{3}^{2}} u_{1} u_{3}
\end{array}\right) d t \\
\gamma_{2}=\int\left(\begin{array}{c}
-a_{1}\left(a_{1}^{2}+a_{1}^{2}+a_{3}^{2}\right) \sqrt{a_{1}^{2}+a_{2}^{2}+a_{3}^{2}+a_{4}^{2}}\left(u_{1}^{2}-u_{2}^{2}-u_{3}^{2}\right) \\
-2 a_{2} a_{3} \sqrt{a_{1}^{2}+a_{2}^{2}+a_{3}^{2}} \sqrt{a_{1}^{2}+a_{2}^{2}+a_{3}^{2}+a_{4}^{2}} u_{1} u_{2}-2 a_{1} a_{4} \sqrt{a_{1}^{2}+a_{2}^{2}} \sqrt{a_{1}^{2}+a_{2}^{2}+a_{3}^{2}} u_{1} u_{3}
\end{array}\right) d t \\
\gamma_{3}=\int\left(2\left(a_{1}^{2}+a_{2}^{2}\right) \sqrt{a_{1}^{2}+a_{2}^{2}+a_{3}^{2}} \sqrt{a_{1}^{2}+a_{2}^{2}+a_{3}^{2}+a_{4}^{2}} u_{1} u_{2}-2 a_{3} a_{4} \sqrt{a_{1}^{2}+a_{2}^{2}} \sqrt{a_{1}^{2}+a_{2}^{2}+a_{3}^{2}} u_{1} u_{3}\right) d t \\
\gamma_{4}=\int\left(2 \sqrt{a_{1}^{2}+a_{2}^{2}}\left(a_{1}^{2}+a_{2}^{2}+a_{3}^{2}\right)^{3 / 2} u_{1} u_{3}\right) d t \\
\sigma=\left(a_{1}^{2}+a_{2}^{2}\right) \sqrt{a_{1}^{2}+a_{2}^{2}+a_{3}^{2}+a_{4}^{2}}\left(a_{1}^{2}+a_{1}^{2}+a_{3}^{2}\right)\left(u_{1}^{2}+u_{2}^{2}+u_{3}^{2}\right)
\end{gathered}
$$

where $u_{1}, u_{2}$ and $u_{3}$ are arbitrary polynomials of parameter "t" and $\sigma(t)=\|\dot{\gamma}(t)\|$.

Proof. Assume that $\gamma: I \rightarrow H^{3}\left(a_{1}, a_{2}, a_{3}, a_{4}\right), \gamma(t)=\left(\gamma_{1}(t), \gamma_{2}(t), \gamma_{3}(t), \gamma_{4}(t)\right)$ be a PH - curve with the speed of a polynomial such as $\sigma(t)$ in $H^{3}\left(a_{1}, a_{2}, a_{3}, a_{4}\right)$. So we have

$$
\left(\gamma_{1}^{\prime}(t)\right)^{2}+\left(\gamma_{2}^{\prime}(t)\right)^{2}+\left(\gamma_{3}^{\prime}(t)\right)^{2}+\left(\gamma_{4}^{\prime}(t)\right)^{2}=\sigma^{2}(t)
$$

Since $\gamma$ lies in $H^{3}\left(a_{1}, a_{2}, a_{3}, a_{4}\right)$ we write

$$
a_{1} \gamma_{1}(t)+a_{2} \gamma_{2}(t)+a_{3} \gamma_{3}(t)+a_{4} \gamma_{4}(t)+a_{5}=0 .
$$


From Equation (19), we get

$$
\left(\frac{\gamma_{1}^{\prime}(t)}{\gamma_{4}^{\prime}(t)}\right)^{2}+\left(\frac{\gamma_{2}^{\prime}(t)}{\gamma_{4}^{\prime}(t)}\right)^{2}+\left(\frac{\gamma_{3}^{\prime}(t)}{\gamma_{4}^{\prime}(t)}\right)^{2}+1=\left(\frac{\sigma(t)}{\gamma_{4}^{\prime}(t)}\right)^{2} .
$$

Differentiating Equation (20), we find

$$
a_{1} \gamma_{1}^{\prime}(t)+a_{2} \gamma_{2}^{\prime}(t)+a_{3} \gamma_{3}^{\prime}(t)+a_{4} \gamma_{4}^{\prime}(t)=0 .
$$

If we make the following substitutions in Equations (21) and (22),

$$
X_{1}=\frac{\gamma_{1}^{\prime}}{\gamma_{4}^{\prime}}, X_{2}=\frac{\gamma_{2}^{\prime}}{\gamma_{4}^{\prime}}, X_{3}=\frac{\gamma_{3}^{\prime}}{\gamma_{4}^{\prime}}, \omega=\frac{\sigma}{\gamma_{4}^{\prime}}
$$

we have

$$
X_{1}^{2}+X_{2}^{2}+X_{3}^{2}+1=\omega^{2}
$$

and

$$
a_{1} X_{1}+a_{2} X_{2}+a_{3} X_{3}+a_{4}=0 .
$$

From Equation (24) $X_{3}$ can be written as

$$
X_{3}=b_{1} X_{1}+b_{2} X_{2}+b_{3}
$$

where $b_{1}=-\frac{a_{1}}{a_{3}}, b_{2}=-\frac{a_{2}}{a_{3}}, b_{3}=-\frac{a_{4}}{a_{3}}$. Considering Equations (23) and (25), we get

$$
\left(1+b_{1}^{2}\right) X_{1}^{2}+\left(1+b_{2}^{2}\right) X_{2}^{2}+2 b_{1} b_{2} X_{1} X_{2}+2 b_{1} b_{3} X_{1}+2 b_{2} b_{3} X_{2}+1+b_{3}^{2}=\omega^{2}
$$

Applying the following transformations in Equation (26)

$$
\begin{aligned}
& X_{1}=Y_{1}-\frac{b_{1} b_{3}}{1+b_{1}^{2}+b_{2}^{2}} \\
& X_{2}=Y_{2}-\frac{b_{2} b_{3}}{1+b_{1}^{2}+b_{2}^{2}}
\end{aligned}
$$

we find

$$
\left(1+b_{1}^{2}\right) Y_{1}^{2}+\left(1+b_{2}^{2}\right) Y_{2}^{2}+2 b_{1} b_{2} Y_{1} Y_{2}+\left(\frac{1+b_{1}^{2}+b_{2}^{2}+b_{3}^{2}}{1+b_{1}^{2}+b_{2}^{2}}\right)=\omega^{2}
$$

We can write this quadratic form as

$$
\left[\begin{array}{ll}
Y_{1} & Y_{2}
\end{array}\right] \cdot\left[\begin{array}{cc}
1+b_{1}^{2} & b_{1} b_{2} \\
b_{1} b_{2} & 1+b_{2}^{2}
\end{array}\right] \cdot\left[\begin{array}{c}
Y_{1} \\
Y_{2}
\end{array}\right]+\left(\frac{1+b_{1}^{2}+b_{2}^{2}+b_{3}^{2}}{1+b_{1}^{2}+b_{2}^{2}}\right)=\omega^{2}
$$

and if we diagonalize Equation (27) with the matrix $Q$ we get

$$
Z_{1}^{2}+\left(\sqrt{1+b_{1}^{2}+b_{2}^{2}} Z_{2}\right)^{2}+\left(\sqrt{\frac{1+b_{1}^{2}+b_{2}^{2}+b_{3}^{2}}{1+b_{1}^{2}+b_{2}^{2}}}\right)^{2}=\omega^{2}
$$

here

$$
Q=\left[\begin{array}{ll}
\frac{-b_{2}}{\sqrt{b_{1}^{2}+b_{2}^{2}}} & \frac{b_{1}}{\sqrt{b_{1}^{2}+b_{2}^{2}}} \\
\frac{b_{1}}{\sqrt{b_{1}^{2}+b_{2}^{2}}} & \frac{b_{2}}{\sqrt{b_{1}^{2}+b_{2}^{2}}}
\end{array}\right]
$$

and satisfies $Z=Q^{-1} Y$. It is seen that Equation (28) is a Pythagorean Quaternary and its solution is

$$
Z_{1}(t)=\frac{r(t)}{s(t)}\left(u_{1}^{2}(t)-u_{2}^{2}(t)-u_{3}^{2}(t)\right)
$$




$$
\begin{gathered}
Z_{2}(t)=\frac{2 r(t)}{\sqrt{1+b_{1}^{2}+b_{2}^{2}} s(t)} u_{1}(t) u_{2}(t) \\
\sqrt{\frac{1+b_{1}^{2}+b_{2}^{2}+b_{3}^{2}}{1+b_{1}^{2}+b_{2}^{2}}}=\frac{2 r(t)}{s(t)} u_{1}(t) u_{3}(t) \\
\omega(t)=\frac{r(t)}{s(t)}\left(u_{1}^{2}(t)+u_{2}^{2}(t)+u_{3}^{2}(t)\right) \\
s(t)=\frac{2 \sqrt{1+b_{1}^{2}+b_{2}^{2}}}{\sqrt{1+b_{1}^{2}+b_{2}^{2}+b_{3}^{2}}} r(t) u_{1}(t) u_{3}(t)
\end{gathered}
$$

where $u_{1}, u_{2}$ and $u_{3}$ are arbitrary polynomials. So, by the backward operations we find $\gamma_{1}, \gamma_{2}, \gamma_{3}$ and $\gamma_{4}$ as declared.

By means of Theorem 3.1, we give the following theorem:

Theorem 4.2. For the hyperplane

$$
H^{3}\left(a_{1}, a_{2}, a_{3}, a_{4}\right)=\left\{\left(p_{1}, p_{2}, p_{3}, p_{4}\right): a_{1} p_{1}+a_{2} p_{2}+a_{3} p_{3}+a_{4} p_{4}+a_{5}=0, a_{1}, a_{2}, a_{3}, a_{4}, a_{5} \in \mathbb{R}\right\}
$$

let $\gamma: I \rightarrow H^{3}, \gamma(t)=\left(\gamma_{1}(t), \gamma_{2}(t), \gamma_{3}(t), \gamma_{4}(t)\right)$ be a PH-curve in $H^{3}$. Then, the curve $\beta: I \rightarrow \mathbb{E}^{4}, \beta(t)=\left(\beta_{1}(t), \beta_{2}(t), \beta_{3}(t), \beta_{4}(t)\right)$ is a PH-helical curve in $\mathbb{E}^{4}$ where

$$
\begin{aligned}
& \beta_{1}=\int\left(\begin{array}{c}
a_{2}\left(a_{1}^{2}+a_{2}^{2}+a_{3}^{2}\right) \sqrt{a_{1}^{2}+a_{2}^{2}+a_{3}^{2}+a_{4}^{2}}\left(u_{1}^{2}-u_{2}^{2}-u_{3}^{2}\right) \\
-2 a_{1} a_{3} \sqrt{a_{1}^{2}+a_{2}^{2}+a_{3}^{2}} \sqrt{a_{1}^{2}+a_{2}^{2}+a_{3}^{2}+a_{4}^{2}} u_{1} u_{2}-2 a_{1} a_{4} \sqrt{a_{1}^{2}+a_{2}^{2}} \sqrt{a_{1}^{2}+a_{2}^{2}+a_{3}^{2}} u_{1} u_{3} \\
+a_{1}\left(a_{1}^{2}+a_{2}^{2}\right)\left(a_{1}^{2}+a_{1}^{2}+a_{3}^{2}\right) \cot \theta\left(u_{1}^{2}+u_{2}^{2}+u_{3}^{2}\right)
\end{array}\right) d t \\
& \beta_{2}=\int\left(\begin{array}{c}
-a_{1}\left(a_{1}^{2}+a_{1}^{2}+a_{3}^{2}\right) \sqrt{a_{1}^{2}+a_{2}^{2}+a_{3}^{2}+a_{4}^{2}}\left(u_{1}^{2}-u_{2}^{2}-u_{3}^{2}\right) \\
-2 a_{2} a_{3} \sqrt{a_{1}^{2}+a_{2}^{2}+a_{3}^{2}} \sqrt{a_{1}^{2}+a_{2}^{2}+a_{3}^{2}+a_{4}^{2}} u_{1} u_{2}-2 a_{1} a_{4} \sqrt{a_{1}^{2}+a_{2}^{2}} \sqrt{a_{1}^{2}+a_{2}^{2}+a_{3}^{2}} u_{1} u_{3} \\
+a_{2}\left(a_{1}^{2}+a_{2}^{2}\right)\left(a_{1}^{2}+a_{1}^{2}+a_{3}^{2}\right) \cot \theta\left(u_{1}^{2}+u_{2}^{2}+u_{3}^{2}\right)
\end{array}\right) d t \\
& \beta_{3}=\int\left(\begin{array}{c}
2\left(a_{1}^{2}+a_{2}^{2}\right) \sqrt{a_{1}^{2}+a_{2}^{2}+a_{3}^{2}} \sqrt{a_{1}^{2}+a_{2}^{2}+a_{3}^{2}+a_{4}^{2}} u_{1} u_{2}-2 a_{3} a_{4} \sqrt{a_{1}^{2}+a_{2}^{2}} \sqrt{a_{1}^{2}+a_{2}^{2}+a_{3}^{2}} u_{1} u_{3} \\
+a_{3}\left(a_{1}^{2}+a_{2}^{2}\right)\left(a_{1}^{2}+a_{1}^{2}+a_{3}^{2}\right) \cot \theta\left(u_{1}^{2}+u_{2}^{2}+u_{3}^{2}\right)
\end{array}\right) d t \\
& \beta_{4}=\int\left(2 \sqrt{a_{1}^{2}+a_{2}^{2}}\left(a_{1}^{2}+a_{2}^{2}+a_{3}^{2}\right)^{3 / 2} u_{1} u_{3}+a_{4}\left(a_{1}^{2}+a_{2}^{2}\right)\left(a_{1}^{2}+a_{1}^{2}+a_{3}^{2}\right) \cot \theta\left(u_{1}^{2}+u_{2}^{2}+u_{3}^{2}\right)\right) d t
\end{aligned}
$$

Hence, the unit axis of the curve $(\beta)$ is

$$
\vec{a}=\left(\frac{a_{1}}{\sqrt{a_{1}^{2}+a_{2}^{2}+a_{3}^{2}+a_{4}^{2}}}, \frac{a_{2}}{\sqrt{a_{1}^{2}+a_{2}^{2}+a_{3}^{2}+a_{4}^{2}}}, \frac{a_{3}}{\sqrt{a_{1}^{2}+a_{2}^{2}+a_{3}^{2}+a_{4}^{2}}}, \frac{a_{4}}{\sqrt{a_{1}^{2}+a_{2}^{2}+a_{3}^{2}+a_{4}^{2}}}\right)
$$

Example 4.3. For the hyperplane $H^{3}(3,4,12,84)$, from Theorem 4.1, it is easy to see that by putting $u_{1}(t)=1, u_{2}(t)=t$ and $u_{3}(t)=1+t$ we obtain a PH-curve $\gamma: I \rightarrow H^{3}, \gamma(t)=\left(\gamma_{1}(t), \gamma_{2}(t), \gamma_{3}(t), \gamma_{4}(t)\right)$ with the followings:

$$
\begin{aligned}
& \gamma_{1}(t)=-\frac{114920}{3} t^{3}-113620 t^{2}-32760 t-\frac{57460}{3} \\
& \gamma_{2}(t)=2830 t^{3}-31785 t^{2}-43680 t+14365 \\
& \gamma_{3}(t)=-37895 t^{2}-131040 t \\
& \gamma_{4}(t)=10985 t^{2}+21970 t
\end{aligned}
$$


We can check that $\|\dot{\gamma}(t)\|=143650\left(t^{2}+t+1\right)$ which is equal to $\sigma(t)$ given in Theorem 4.1 as well. Also by the way of Theorem 4.2, assuming $\cot \theta=1$ we get a PH-helix curve from the curve $\gamma$ such as $\beta: I \rightarrow \mathbb{E}^{4}, \beta(t)=\left(\beta_{1}(t), \beta_{2}(t), \beta_{3}(t), \beta_{4}(t)\right)$ with the followings:

$$
\begin{aligned}
& \beta_{1}(t)=-\frac{109850}{3} t^{3}-111085 t^{2}-27690 t-\frac{54925}{3} \\
& \beta_{2}(t)=\frac{92950}{3} t^{3}-28405 t^{2}-36920 t+\frac{46475}{3} \\
& \beta_{3}(t)=6760 t^{3}-27755 t^{2}-110760 t+3380 \\
& \beta_{4}(t)=47320 t^{3}+81965 t^{2}+163930 t+23660
\end{aligned}
$$

Furthermore it can be calculated that $\|\dot{\beta}(t)\|=143650 \sqrt{2}\left(t^{2}+t+1\right)$ that means $\beta$ is also a PH-curve. At the same time, since $\langle\dot{\beta}(t), \vec{a}\rangle=\frac{85 \sqrt{2}}{2}$ for the constant vector $\vec{a}=\left(\frac{3}{85}, \frac{4}{85}, \frac{12}{85}, \frac{84}{85}\right)$, it's seen that $\beta$ is a helical curve in $\mathbb{E}^{4}$.

Example 4.4. $\gamma: I \rightarrow H^{4}(3,12,4,84,132), \gamma(t)=\left(\gamma_{1}(t), \gamma_{2}(t), \gamma_{3}(t), \gamma_{4}(t), \gamma_{5}(t)\right)$ is a PH-curve components below:

$$
\begin{aligned}
& \gamma_{1}(t)=-58984900 t^{3}-55462500 t^{2}-96990100 t \\
& \gamma_{2}(t)=-30149075 t^{2}-152020800 t \\
& \gamma_{3}(t)=44238675 t^{3}-73950000 t^{2}-6434925 t \\
& \gamma_{4}(t)=72537725 t^{3}-99969350 t \\
& \gamma_{5}(t)=-39918125 t^{2}+79836250 t
\end{aligned}
$$

It can be calculated that $\|\dot{\gamma}(t)\|=221193375\left(t^{2}+1\right)$. Also the curve $\beta$ which is obtained from $\gamma$ by using Theorem 3.2 is a PH-helical curve in $\mathbb{E}^{5}$ with the followings

$$
\begin{aligned}
& \beta_{1}(t)=-\frac{231712975}{4} t^{3}-55462500 t^{2}-\frac{375280525}{4} t \\
& \beta_{2}(t)=4226625 t^{3}-30149075 t^{2}-139340925 t \\
& \beta_{3}(t)=45647550 t^{3}-73950000 t^{2}-2208300 t \\
& \beta_{4}(t)=29586375 t^{3}+72537725 t^{2}-11210225 t \\
& \beta_{5}(t)=46492875 t^{3}-39918125 t^{2}+219314875 t
\end{aligned}
$$

Furthermore it can be calculated that $\|\dot{\beta}(t)\|=\frac{1105966875}{4}\left(t^{2}+1\right)$ that means $\beta$ is also a PH-curve. At the same time, since $\langle\dot{\beta}(t), \vec{a}\rangle=\frac{471}{5}$ for the constant vector $\vec{a}=\left(\frac{3}{157}, \frac{12}{157}, \frac{4}{157}, \frac{84}{157}, \frac{132}{157}\right)$, it's seen that $\beta$ is a helical curve in $\mathbb{E}^{5}$.

\section{Conclusion}

In this paper, we give a method to obtain PH-curves from arbitrary planar curves. And then we show that there are PH-helical curves which are obtained from PH-curves that we constructed. We give the derivation of the method in $\mathbb{E}^{4}$ as an application in details and another PH-helical curve in $\mathbb{E}^{5}$ without detailed computations. Changing the coefficients and the polynomials used in examples, one can obtain any PH-helical curves whose components are of any degrees. Of course, it's possible to extend Theorem 4.1 and and 4.2 to any dimension of $\mathbb{E}^{n+1}$. In addition, because we consider only the rational solutions of $(n+1)$-tuples of Pythagorean equation, we can say that it may not be possible to obtain all PH-helical curves with our method given in the paper. So this is an open problem in the literature. 


\section{Author Contributions}

All the authors contributed equally to this work. They all read and approved the final version of the manuscript.

\section{Conflicts of Interest}

The authors declare no conflict of interest.

\section{Acknowledgment}

This work was supported by the Office of Scientific Research Projects Coordination at Çanakkale Onsekiz Mart University, Grant number: FHD-2020-3452.

\section{References}

[1] M. A. Lancret, $M$ ' emoire sur les courbes 'a double courbure, M'emoires pr'esent'es 'a l'Institut des Sciences, Letters et arts par divers savants, Tome 1(1802) 416-454.

[2] W. Kuhnel, Differential Geometry: Curves-Surfaces-Manifolds, Braunchweig, Friedr. Vieweg \& Sohn, 1999.

[3] R. T. Farouki, T. Sakkalis, Pythagorean Hodographs, IBM Journal of Research and Development 34(5) (1999) 736-752.

[4] R. T. Farouki, T. Sakkalis, Pythagorean-Hodograph Space Curves, Advances in Computational Mathematics 2(1) (1994) 41-66.

[5] R. T. Farouki, C. Y. Han., C. Manni, A. Sestini, Characterization and Construction of Helical Polynomial Space Curves, Journal of Computational and Applied Mathematics 162(2) (2004) 365-392.

[6] R. T. Farouki, Pythagorean-Hodograph Curves: Algebra and Geometry Inseparable, Springer, Berlin, Heidelberg, 2008.

[7] R. T. Farouki, C. Giannelli, A. Sestini, Helical Polynomial Curves and Double Pythagorean Hodographs I Quaternion and Hopf map representations, Journal of Symbolic Computation 44(2) (2009) 161-179.

[8] R. T. Farouki, C. Giannelli, A. Sestini, Helical Polynomial Curves and Double Pythagorean Hodographs II. Enumeration of Low-Degree Durves, Journal of Symbolic Computation 44(4) (2009) 307-332.

[9] R. T. Farouki, M. Al-Kandari, T. Sakkalis, Structural Invariance of Spatial Pythagorean Hodographs, Computer Aided Geometric Design 19(6) (2002) 395-407.

[10] R. T. Farouki, Z. Sír, Rational Pythagorean-Hodograph Space Curves, Computer Aided Geometric Design 28(2) (2011) 78-88.

[11] H. Pottman, Curve Design with Rational Pythagorean-Hodograph Curves, Advances in Computational Mathematics 3 (1995) 147-170.

[12] S. Izumiya, N. Takeuchi, Generic Properties of Helices and Bertrand Curves, Journal of Geometry 74 (2002) 97-109.

[13] Ç. Camcı K. İlarslan, A New Method for Construction of PH-Helical Curves in $\mathbb{E}^{3}$, Comptes Rendus De L Academie Bulgare Des Sciences, 72(3) (2019) 301-308. 
[14] H. Gluck, Higher Curvatures of Curves in Euclidean Space, The American Mathematical Monthly 37 (1966) 699-704.

[15] M. C. Romero-Fuster, E. Sanabria-Codesal, Generalized Helices Twistings and Flattenings of Curves in n-Space, Matematica Contemporanea 17 (1999) 267-280.

[16] E. Ödamar, H. H. Hacısalihoğlu, A Characterization of Inclined Curves in Euclidean n-Space, Communications Faculty of Sciences University of Ankara Series A1-Mathematics and Statistics 24 (1975) 15-23.

[17] Ç. Camcı K. İlarslan, L. Kula, H. H. Hacısalihoğlu, Harmonic Cuvature and General Helices, Chaos Solitons Fractals 40 (2009) 2590-2596.

[18] K. K. Kubota, Pythagorean Triples in Unique Factorization Domains, The American Mathematical Monthly 79 (1972) 503-505. 